\title{
Einfluß von Lagerung und Temperatur auf die Analyse von Steroiden in Plasma und Blut
}

\author{
Von H.K. Kley ${ }^{1}$ ) \\ Medizinische Klinik C und Poliklinik, Universität Düsseldorf und \\ W. Rick \\ Institut für Klinische Chemie und Laboratoriumsdiagnostik, Universität Düsseldorf
}

(Eingegangen am 1. Oktober 1983/19. Januar 1984)

Zusammenfassung: Wir untersuchten den Einfluß der Lagerung auf Blut- und Plasmaproben, der Aufbewahrung bei verschiedenen Temperaturen sowie 10maligen Auftauens und Einfrierens auf die Analyse folgender Steroide: Cortisol, Aldosteron, 17-Hydroxyprogesteron, Testosteron, Androstendion, Dehydroepiandrosteronsulfat, Östron und Östradiol sowie Sexualhormone bindendes Globulin (SHBG) und die Bindung von Cortisol und Testosteron im Plasma.

Zehnmaliges Einfrieren und Auftauen beeinflußte die Analysenergebnisse der Steroide und ihre Plasmabindung nicht, so daß Mehrfachanalysen zu verschiedenen Zeiten aus einer Plasmaprobe möglich sind. Die Lagerung von Plasmaproben bei Raum- oder Kühlschranktemperatur $\left(22^{\circ} \mathrm{C}\right.$ bzw. $\left.4^{\circ} \mathrm{C}\right)$ über 4 Tage erbrachte gegenüber einer Lagerung bei $-28^{\circ} \mathrm{C}$ keine entscheidenden Nachteile im Analysenergebnis. Allein bei Östron, Östradiol, Aldosteron und Androstendion kam es zu einem Abfall der Mittelwerte, die jedoch alle im 2 SD-Bereich der Analysenvarianż lagen. Auch SHBG und die Bindung von Cortisol und Testosteron blieben durch diese Art der Vorbehandlung unbeeinflußt. Durch die Lagerung der Proben als Vollblut bei $4{ }^{\circ} \mathrm{C}$ oder $22^{\circ} \mathrm{C}$ waren Abweichungen in den meisten Analysenergebnissen nachweisbar; diese waren jedoch relativ gering $(<10 \%)$. Allein Aldosteron (12\%) und Androstendion (14,5\%) wiesen einen nennenswerten Abfall auf. Dagegen führte die Lagerung als Vollblut bei $22^{\circ} \mathrm{C}$ zu einer deutlichen Änderung der Steroidbindung, die für Testosteron $26 \%$ und für Cortisol $15 \%$ betrug.

Aus den Untersuchungen folgt, daß̣ Steroide und ihre Plasmabindung in Blut oder Plasma gegenüber den meisten üblichen äußeren Einflüssen relativ stabil sind ụnd auch aus weniger sorgfältig behandelten Proben (Postversand) in dèr Règel noch ausreichend zuverlässig analysiert werden können.

Influence of storage and temperature on steroid analysis in plasma and blood

Summary: In the analysis of steroid hormones careful attention is usually paid to blood collection and plasma storage. However, the appropriate care of samples cannot always be assured in routine work with steroids. Therefore, the stability of cortisol, aldosterone, 17-hydroxyprogesterone, testosterone, androstenedione, dehydroepiandrosteronesulphate, oestrone, oestradiol, sex hormone binding globulin (SHBG) and, the binding of testosterone and cortisol to plasma proteins in blood and plasma were studied before and after various handling procedures.

Ten cycles of alternate freezing and thawing of plasma did not significantly affect the levels of the steroids or their plasma binding. The greatest differences, compared with controls, were seen for aldosterone $(-6.2 \%)$ and oestradiol $(-5.3 \%)$. Plasma storage at $-28^{\circ} \mathrm{C}$ was hardly superior to a 4 days storage at $4{ }^{\circ} \mathrm{C}$ (refrigera-

') Unterstützt durch die Deutsche Forschungsgemeinschaft (KI 346). 
tor) or $22{ }^{\circ} \mathrm{C}$ (room temperature). Although androstenedione $(-10.9 \%)$, oestrone $(-10.2 \%)$ and oestradiol $(-12.2 \%)$ levels decreased by more than $10 \%$, the means of all analyses were still in the 2 SD range. Even SHBG and the steroid binding were only slightly affected by temperature. When whole blood was stored at $4^{\circ} \mathrm{C}$ or $22^{\circ} \mathrm{C}$, the resulting values differed from those obtained with plasma, but the differences were usually less than $10 \%$. Although the levels were within the 2 SD range, whole blood showed a decrease of $12.3 \%$ for aldosterone and $14.5 \%$ for androstenedione. In contrast, plasma binding of testosterone $(25.9 \%)$ and cortisol $(15.1 \%)$ were substantially affected by storage at $22^{\circ} \mathrm{C}$ in whole blood.

It is concluded that repeated freezing and thawing of plasma, or storage at various temperatures have only a small effect on the measured levels of steroids and their plasma binding. Although it is not advisable, even whole blood may be used for the analysis of steroid concentrations.

\section{Einfiihrung}

Bei Durchsicht der methodischen Abschnitte wissenschaftlicher Publikationen findet man kaum eine Arbeit über Steroidhormone, in der nicht auf die besondere Sorgfalt der Probennahme und -behandlung eingegangen wird. Diese Sorgfalt kann bei wissenschaftlichen Untersuchungen eingehalten werden, ist jedoch bei vielen Routineuntersuchungen nicht immer gewährleistet und stellt in einem Labor, das auch eingesandte Proben analysiert, eher die Ausnahme dar. Diese ,präanalytische Sorgfalt zur Elimination von Störfaktoren" $(1,2,3)$ scheint auch erforderlich zu sein, wenn man die vielen Möglichkeiten einer Änderung der Molekülstruktur von Steroiden und ihrer Plasmabindung vermeiden will: Enzyme im Plasma sind nämlich in der Lage, Proteine abzubauen $(4,5)$, Erythrocyten können Steroide binden (6) und metabolisieren $(7,8,9)$; Lymphocyten und Granulocyten binden Steroidhormone durch intrazelluläre Rezeptoren (10), und es sind eine Vielzahl von Bakterien bekannt, die Steroide zu verändern vermögen $(11,12)$. Dies gilt in noch stärkerem Maße, wenn man berücksichtigt, daß einige der am häufigsten gemessenen Steroidhormone an Plasmaproteine gebunden sind (13), die gegenüber Veränderungen durch Enzyme, Temperatur oder Bakterien besonders empfindlich zu sein scheinen (4). Aus diesem Grunde ist es verwunderlich, daß bisher nur wenige Publikationen vorhanden sind $(14,15,16)$, die meist für einzelne Steroide versuchten, das Ausmaß solcher präanalytischer Störfaktoren zu bestimmen.

Wir untersuchten deshalb den Einfluß von Lagerung, von Temperatur $\left(4^{\circ} \mathrm{C}\right.$ und $\left.22^{\circ} \mathrm{C}\right)$ und mehrfachem Auftauen auf die Konzentration der wichtigsten, in unserem Labor gemessenen Steroide. Da bei extremen Hormonwerten sich oft die Frage nach dem freien Hormonanteil (bzw. der Konzentration an Bindungsprotein) stellt, wurden die Proben vor Messung von freiem Cortisol, freiem Testosteron und Sexualhormone bindenden Globulin (SHBG) dem gleichen Verfahren der präanalytischen Probenbehandlung unterzogen.

\section{Methodik}

Untersucht wurde das Blut nüchterner männlicher Patienten, die wegen Hämochromatose regelmäßig eine Aderlaßtherapie erhielten. Außer der Hämochromatose lagen entsprechend den klinischen und klinisch-chemischen Kenngrößen keine weiteren $\mathrm{Er}$ krankungen vor. Die Leberfunktion war in allen Fällen normal, und ein Diabetes mellitus bestand nicht. Etwa $500 \mathrm{ml}$ Blut wurde als Aderlaß zwischen 8.00 und 9.00 Uhr einer Kubitalvene entnommen, sofort heparinisiert und für jedes einzelne Steroid in jeweils 6 mal 6 Proben aufgeteilt. Jeweils 6 dieser Proben wurden vor Analyse einer gleichartigen Behandlung unterzogen:

1. Sechs Plasmaproben wurden nach Zentrifugation sofort bei $-28^{\circ} \mathrm{C}$ eingefroren und dienten als Kontrollen.

2. Sechs Plasmaproben wurden bèi $=28^{\circ} \mathrm{C}$ eingefroren und $24 \mathrm{~h}$ später bei Raumtemperatur $\left(22^{\circ} \mathrm{C}\right)$ über $8 \mathrm{~h}$ aufgetaut. Dieser Vorgang des Einfrierens und Auftauens wurde $10 \mathrm{mal}$ wiederholt, bevor eine Steroidanalyse erfolgte.

3. Sechs Plasmaproben wurden 4 Tage lang bei $4^{\circ} \mathrm{C}$ gelagert, und 4. sechs Plasmaproben lagerten über 4 Tage bei $22^{\circ} \mathrm{C}$.

5. Sechs Proben mit Vollblut wurden ebenfalls über 4 Tage bei $4^{\circ} \mathrm{C}$ belassen bevor sie zentrifugiert wurden.

6. Sechs Proben mit Vollblut wurden 4 Tage lang bei $22^{\circ} \mathrm{C}$ vor Abtrennung des Plasmas gelagert.

Nach dieser präanalytischen Behandlung lagerten alle Proben bei $-28^{\circ} \mathrm{C}$ bis zur Analyse der einzelnen Steroide.

Probengewinnung und -aufteilung erfolgte in Plastikgefäßen ohne spezielle Vorsicht bezüglich Sterilität, so daß weitgehend Laboratoriumsroutine-Bedingungen vorlagen. Uim Verdunstung zu vermeiden, wurden die Probengefäße bis zur Analyse sicher verschlossen gehalten.

Die Steroide wurden radioimmunologisch unter Verwendung spezifischer Antikörper gemessen. Außer Dehydroepiandrosteronsulfat und in einem Zusatzversuch Testosteron wurden alle Steroide vor Analyse chromatographiert. Alle Analysen werden in unserem Labor routinemäßig durchgeführt. Bei der Messung von Dehydroepiandrosteronsulfat und Testosteron (ohne Chromatographie) wurden käufliche Kits verwendet (Bio-Merieux, Nörtingen), während die übrigen Steroide mit èigenen Antikörpern nach chromatographischer Reinigung gemessen wurden: Cortisol (17), 
Aldosteron (18) 17-Hydroxyprogesteron (19), Ostron, Ostradiol (20), Testosteron und Androstendion (21). Des weiteren wurde mit Hilfe der Gleichgewichtsdialyse freies Cortisol und freies Testosteron gemessen (17). SHBG wurde durch Ammoniumsulfatpräzipitation und mit Dihydrotestosteron als Marker bestimmt (22).

Die Sensitivität der Messung von Steroiden betrug 5-11 pg pro Ansatz. Die Variationen der Bestimmung von Tag zu Tag (interassay variation) wurden im Verlaufe von $1-5$ Jahren ermittelt. Sie betrugen in der Regel weniger als $10 \%$, im einzelnen: Cortisol $(3,8 \%)$, Aldosteron $(8,7 \%), 17$-Hydroxyprogesteron $(5,5 \%)$, Testosteron $(4,4 \%)$, Testosteron ohne Chromatographie $(3,8 \%)$, Androstendion $(8,1 \%)$, Dehydroepiandrosteronsulfat $(5,0 \%)$, Ostron $(5,3 \%)$, Ostradiol $(6,4 \%)$, SHBG $(4,7 \%)$, freies Testosteron $(12,4 \%)$, freies Cortisol $(8,9 \%)$. Es wurde versucht, alle Bestimmungen in möglichst wenigen Assays durchzuführen. Mitgelaufene Standards sicherten die Vergleichbarkeit der einzelnen Meßserien. Die Ergebnisse sind angegeben als $\bar{x} \pm$ SD. Student's t-Test wurde zur statistischen Analyse verwendet.
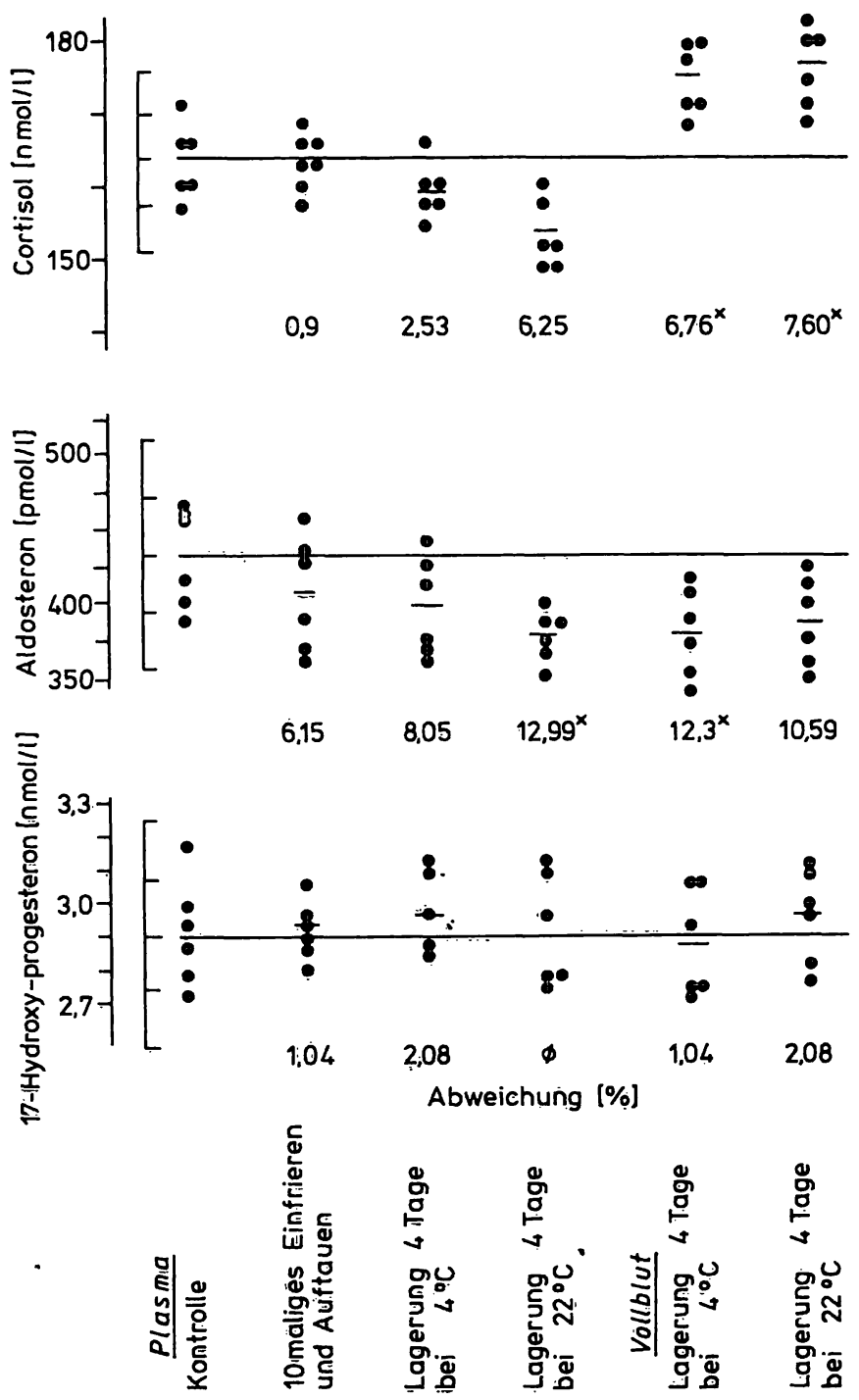

Abb. 1. Stabilität von Cortisol, Aldosteron und 17-Hydroxyprogesteron in Plasma und Blut. Der Varianzbereich, ausgedrückt als 2SD-Bereich bei Messung von Tag zu Tag, bezogen auf den Mittelwert der Kontrollen, ist neben der Abszisse eingetragen. Die Abweichung (\%) der Mittelwerte der einzelnen Plasma- und Blutserien von den Kontrollen ist eingezeichnet.

$x: p<0,05, x x: p<0,01$ bei Vergleich der behandelten Proben mit den Kontrollen.

\section{Ergebnisse}

Die gemessenen Konzentrationen der Steroide in den Kontrollplasmen lagen im Bereich gesunder männlicher Probanden mit Ausnahme von Testosteron, das bei Patienten mit Hämochromatose erniedrigt gefunden wird (23).

Einfluß wiederholten Einfrierens und Auftauens

\section{Zehnmaliges Einfrieren und Auftauen}

Zehnmaliges Einfrieren (bei $-28^{\circ} \mathrm{C}$ ) und zehnmaliges Auftauen über $8 \mathrm{~h}$ (bei $22^{\circ} \mathrm{C}$ ) änderte die Konzentrationen der Steroide im Plasma nicht signifikant; alle Werte liegen im 1 SD-Bereich (Abb. 14). Auch im direkten Vergleich mit den Kontrollen war für kein Steroid ein signifikanter Unterschied aufdeckbar. Das gleiche gilt bei Bestimmung der freien Steroide von Cortisol und Testosteron oder des Bindungsproteins SHBG, deren Werte fast identisch mit den Kontrollen sind. Die Abbildungen zeigen jedoch auch, daß Aldosteron, Androstendion, Dehydroepiandrosteronsulfat, Östron, Östradiol und freies Testosteron einen leichten - nicht signifikanten - Abfall von etwa 5\% (4,3-6,2\%) aufweisen.

Einfluß der Lagerung von Plasmaproben bei verschiedenen Temperaturen

\section{Lagerung bei $4^{\circ} \mathrm{C}$}

Bei Lagerung der Plasmaproben über 4 Tage bei $4^{\circ} \mathrm{C}$ weisen Östron, Östradiol und das freie Testosteron eine signifikante Abnahme auf. Ähnlich empfindlich reagierte Aldosteron (Abfall von 8,1\%) und das Androstendion (Abnahme von 7,3\%), ohne daß hier jedoch die (intraassay) Varianz der Meßmethode unterschritten wurde. Die Abweichung der übrigen Steroide von den Kontrollwerten war minimal und lag zwischen $0,7 \%$ (Dehydroepiandrosteronsulfat) und $2,5 \%$ (Cortisol). Auffällig ist die größere Abweichung von Testosteron (6,0\%) gegenüber der des direkt gemessenen Testosterons ohne Chromatographie.

\section{Lagerung bei $22^{\circ} \mathrm{C}$}

Bei Lagerung der Plasmaproben über 4 Tage bei $22{ }^{\circ} \mathrm{C}$ war bei den meisten Steroiden der Abfall stärker ausgeprägt als bei der niedrigeren Temperatur von $4^{\circ} \mathrm{C}$ (Abb. 1-4). Der Unterschied zu den Kontrollen war signifikant $(p<0,05)$ für Aldosteron, Testosteron, Androstendion, Óstron, Östradiol und 

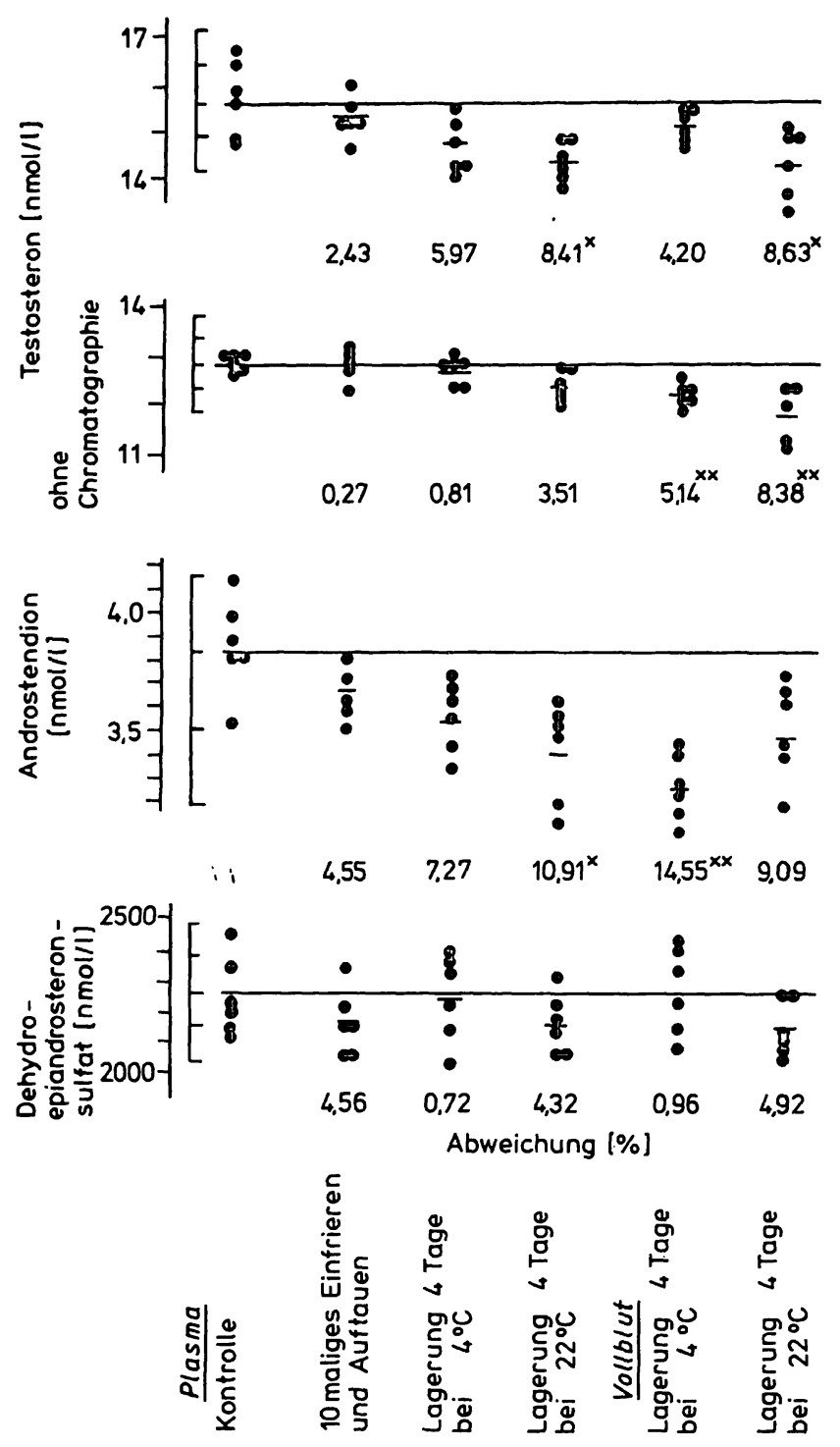

Abb. 2. Stabilität von Testosteron, Androstendion und Dehydroepiandrosteronsulfat. Testosteron wurde einmal mit und einmal ohne chromatographische Reinigung gemessen. Weitere Angaben s. Legende Abb. 1.

freies Testosteron. Auffällig war, daß SHBG und freies Cortisol relativ gering von dieser Lagerung bei $4{ }^{\circ} \mathrm{C}$ bzw. $22^{\circ} \mathrm{C}$ beeinflußt wurden. Die Differenz zu den Kontrollen betrug nur 1,0\% (SHBG) bzw. 2,3\% (freies Cortisol).

Einfluß der Lagerung von Vollblut bei verschiedenen Temperaturen

\section{Lagerung bei $4^{\circ} \mathrm{C}$}

Die Lagerung von Vollblut bei $4^{\circ} \mathrm{C}$ über 4 Tage führte nur zu geringen Veränderungen der Konzentrationen von 17-Hydroxyprogesteron (1\%), Östron $(4,1 \%)$, Testosteron $(5,1 \%)$, Dehydroepiandrosteronsulfat $(1 \%)$ und den freien Steroiden, während
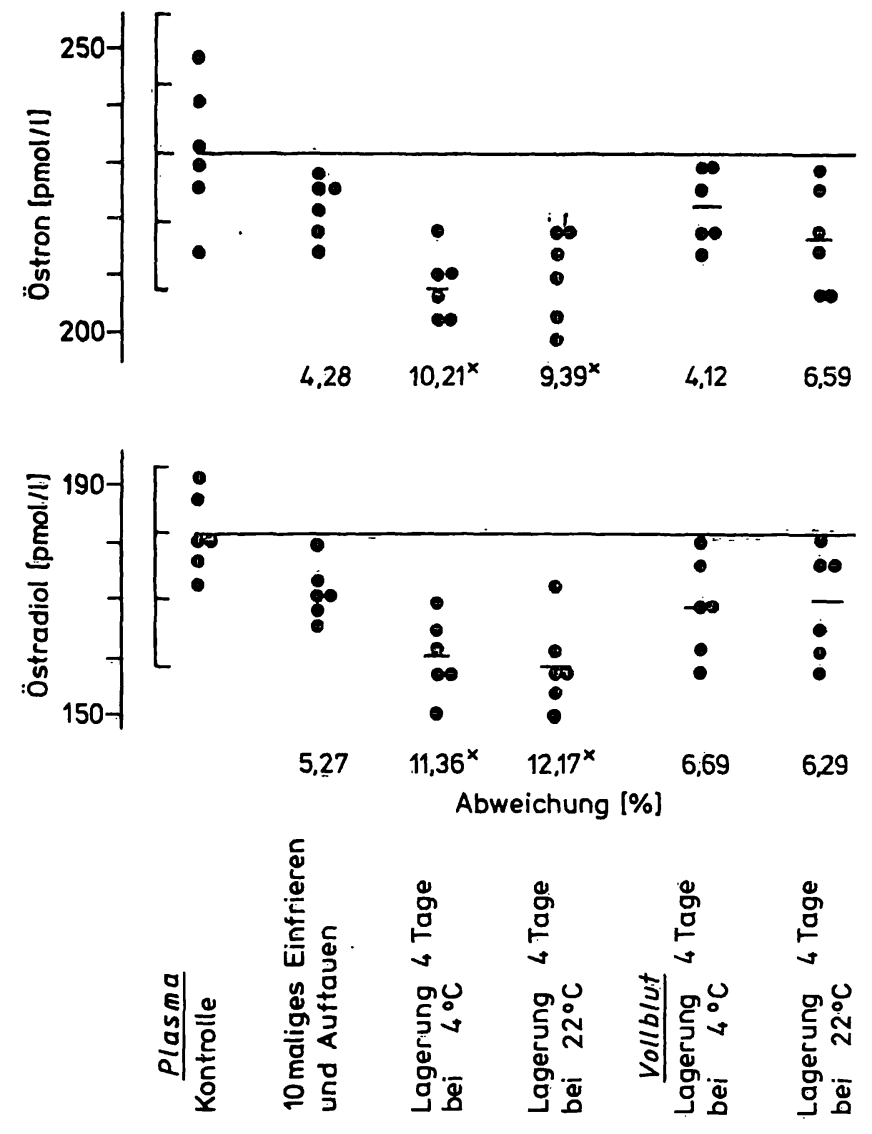

Abb. 3. Der Einfluß verschiedener präanalytischer Störfaktoren auf die Konzentrationen von Östron und Östradiol. Weitere Angaben s. Legende Abb. 1.

Aldosteron (12,3\%) und Androstendion (14,5\%) deutlicher betroffen waren $(p<0,05)$. SHBG zeigte keine signifikante Beeinträchtigung durch diese Vorbehandlung $(4,9 \%)$. Cortisol war gegenüber den Kontrollen gering höher $(6,8 \%)$.

\section{Lagerung bei $22^{\circ} \mathrm{C}$}

Nach 4-tägiger Lagerung der Blutproben bei Raumtemperatur $\left(22^{\circ} \mathrm{C}\right)$ fanden sich die größten Abweichungen für das freie Cortisol und das freie Testosteron $(15,1 \%$ und $25,9 \%)$, die mit nachlassender Bindung an Plasmaproteine deutlich zunahmen. SHBG war durch diese Vorbehandlung wenig beeinflu $\beta t$ $(4,4 \%)$. Gegenüber den gleichartig behandelten Plasmaproben fand sich kein signifikanter Unterschied mit Ausnahme von Cortisol, das wiederum eine leicht höhere Konzentration aufwies. Betroffen waren vorwiegend Aldosteron $(10 ; 6 \%)$, Östron $(6,6 \%)$, Óstradiol $(6 ; 3 \%)$, Testosteron $(8,6 \%)$, Androstendion $(9,1 \%)$, während 17-Hydroxyprogesteron, Dehydroepiandrosteronfulfat und SHBG nur um $2,1-4,9 \%$ abwichen. 

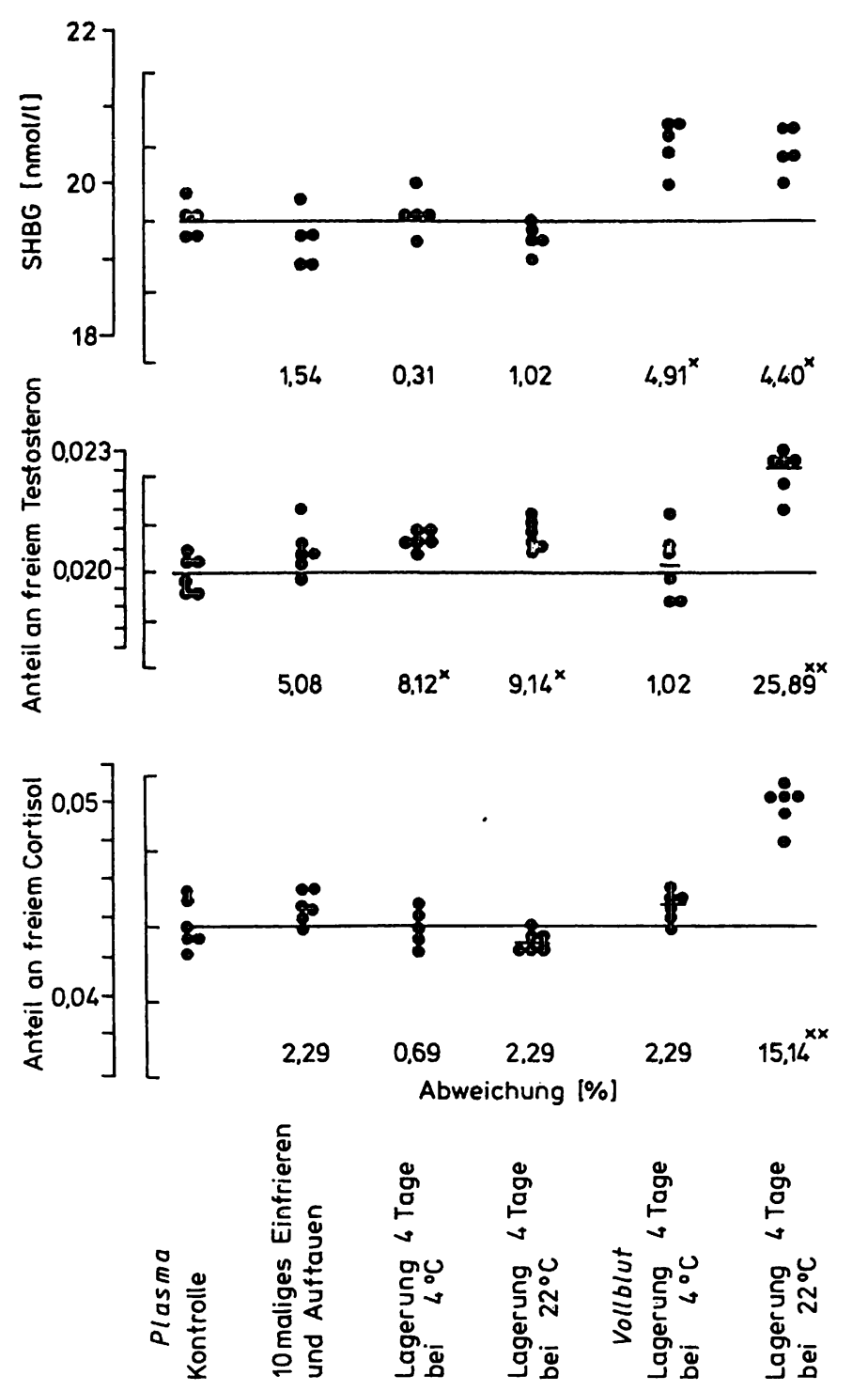

Abb. 4. Stabilität von Bindungsproteinen im Plasma. Gemessen wurde SHBG (Sexualhormone bindendes Globulin) sowie das freie Testosteron und das freie Cortisol nach unterschiedlicher Vorbehandlung der Plasma- bzw. Blutproben. Weitere Angaben s. Legende Abb. 1.

Bestimmt man die durchschnittliche Abweichung der Steroidkonzentration von den Kontrollen, hervorgerufen durch die hier untersuchten Störfaktoren (Tab. 1), dann bewirkt mehrfaches Auftauen und Einfrieren der Plasmaproben die geringste Veränderùng, und ist der „größte“ Störfaktor die Lagerung bei $22^{\circ} \mathrm{C}$ (Raumtemperatur). Dabei ergeben sich keine deutlichen Unterschiede, ob die Proben als Blut oder Plasma gelagert wurden, Anders ist es jedoch bei den Bindungskenngrößnen freies Testosteron und freies Cortisol, wo Lagerung als Vollblut über 4 Tage bei $22^{\circ} \mathrm{C}$ zu erheblichen Abweichungen führte. Aus Tabelle 1 folgt außerdem, daß eine gewisse „Hierarchie“ für die Stabilität der Steroide aufgestellt werden kann. Danach sind Aldosteron, Androstendion und die Óstrogene besonders emp- findlich, während 17-Hydroxyprogesteron, Dehydroepiandrosteronsulfat, Cortisol und Testosteron für die gewählten Störfaktoren relativ stabil sind.

Insgesamt fanden sich nur wenige Einzelproben und keine Mittelwerte, die in ihren Konzentrationen auBerhalb des 2SD-Bereichs der Analysenvarianz lagen.

\section{Diskussion}

Die Untersuchungen bestätigen einige frühere Einzelbefunde $(14,15,16,24)$ und zeigen, daß auch weniger sorgfältig behandelte Proben hinreichend zuverlässig auf Steroide analysiert werden können.

Sowohl bei wissenschaftlichen als auch bei Routineuntersuchungen ist es gelegentlich notwendig, eine Analyse zu wiederholen bzw. ein weiteres Hormon aus der gleichen Plasmaprobe zu messen. Wie die Versuche mit zehnmaligem Auftauen und Einfrieren zeigen, ist dies möglich, ohne einen entscheidenden Fehler in der Analyse von Steroiden zu riskieren (14).

Da Plasmaproben häufig mit der Post gesendet werden - teilweise in Eis verpackt - untersuchten wir den Effekt der Temperatur auf die Stabilität von Steroiden. Es wurde ein Zeitraum von 4 Tagen gewählt, da dieser auch bei Postsendungen selten überschritten wird. Diese Art der Lagerung führte zu einem Abfall der meisten gemessenen Steroidkonzentrationen. Er beträgt bei $4{ }^{\circ} \mathrm{C}$ jedoch nur etwa $4,8 \%$ $(0,8-11,4 \%)$ und bei $22{ }^{\circ} \mathrm{C} 6,7 \%(0-12,2 \%)$. Die Abweichung liegt in allen Fällen noch im 2SD-Bereich für die Messung der einzelnen Steroide. Dieser Fehler ist für wissenschaftliche Fragen nicht mehr tolerabel, ist jedoch bei der Interpretation der meisten klinischen Untersuchungen von geringerer Relevanz.

Gelegentlich werden statt Plasma Blutproben zugesendet, oder eine Zentrifugation der Probe ist während der Nacht oder am Wochenende nicht sofort möglich. Wie die Untersuchungen zeigen, ist es bei den meisten Steroiden gleichgültig, ob die Proben als Plasma- oder Blutproben gelagert wurden, liegt doch die Abweichung der Steroidkonzentrationen etwa in der gleichen Größenordnung. Eine Hämolyse war in den Blutproben nicht nachweisbar, jedoch dürfte auch dies keinen zusätzlichen Fehler hervorrufen (16). Auch bei Vollblut zeigte sich, daß die Lagerung bei $4^{\circ} \mathrm{C}$ gegenüber der bei $22^{\circ} \mathrm{C}$ keinen entscheidenden Vorteil lieferte. Bei der Lagerung als Vollblut fanden sich die größten Abweichungen zu den Kontrollen für Aldosteron (Abfall maximal bis 
Tab. 1. Abweichung der Mittelwerte der Meßdaten aus Abbildung 1-4 von den Kontrollen in Abhängigkeit von den ,präanalytischen Störfaktoren" $\left(\bar{x}_{1}\right)$ bzw. den gemessenen Steroiden $\left(\bar{x}_{2}\right)$. Im unteren Teil der Tabelle sind die Werte für die Steroidbindung und das Bindungsprotein SHBG angegeben.

Entsprechend dieser Synopsis sind die „empfindlichsten“ Steroide Aldosteron, Androstendion, Ostradiol und Ostron und der "größte“" Störfaktor für Steroide und deren Bindung die Lagerung von Plasma oder Blut über 4 Tage bei Raumtemperatur.

\begin{tabular}{|c|c|c|c|c|c|c|}
\hline \multirow[t]{2}{*}{ Kenngröße } & \multicolumn{3}{|l|}{ Plasma } & \multirow{2}{*}{$\begin{array}{l}\text { Blut } \\
\text { Lagerung } \\
4 \text { Tage } \\
\text { bei } 4^{\circ} \mathrm{C}\end{array}$} & \multirow{2}{*}{$\begin{array}{l}\because 1 \\
\text { Lagerung } \\
4 \text { Tage } \\
\text { bei } 22^{\circ} \mathrm{C}\end{array}$} & \multirow[b]{2}{*}{$\overline{\mathbf{x}}_{2}$} \\
\hline & $\begin{array}{l}\text { Zehnmaliges } \\
\text { Einfrieren und } \\
\text { Auftauen }\end{array}$ & $\begin{array}{l}\text { Lagerung } \\
4 \text { Tage } \\
\text { bei } 4^{\circ} \mathrm{C}\end{array}$ & $\begin{array}{l}\text { Lagerung } \\
4 \text { Tage } \\
\text { bei } 22^{\circ} \mathrm{C}\end{array}$ & & & \\
\hline Cortisol & 0,90 & 2,53 & 6,25 & 6,76 & 7,60 & 4,81 \\
\hline Aldosteron & 6,15 & 8,05 & 12,99 & 12,30 & 10,59 & 10,02 \\
\hline 17-Hydroxyprogesteron & 1,04 & 2,08 & 0,00 & 1,04 & 2,08 & 1,25 \\
\hline Testosteron & 2,43 & 5,97 & 8,41 & 4,20 & 8,63 & 5,93 \\
\hline Testosteron ohne Chromatographie & 0,27 & 0,81 & 3,51 & 5,14 & 8,38 & 3,62 \\
\hline Androstendion & 4,55 & 7,27 & 10,91 & 14,55 & 9,09 & 9,27 \\
\hline Dehydroepiandrosteronsulfat & 4,56 & 0,72 & 4,32 & 0,96 & 4,92 & 3,10 \\
\hline Ostron & 4,28 & 10,21 & 9,39 & 4,12 & 6,59 & 6,92 \\
\hline \multirow[t]{2}{*}{ Óstradiol } & 5,27 & 11,36 & 12,17 & 6,69 & 6,29 & 8,36 \\
\hline & 3,27 & 5,44 & 7,55 & 6,20 & 7,13 & 5,92 \\
\hline SHBG & 1,54 & 0,31 & 1,02 & 4,91 & 4,40 & 2,44 \\
\hline Freies Testosteron : & 5,08 & 8,12 & 9,14 & 1,02 & 25,89 & 9,85 \\
\hline \multirow[t]{2}{*}{ Freies Cortisol } & 2,29 & 0,69 & 2,29 & 2,29 & 15,14 & 4,54 \\
\hline & 2,97 & 3,04 & 4,15 & 2,73 & 25,14 & 5,61 \\
\hline
\end{tabular}

$12,3 \%$ ) und Androstendion (14,55\%). Diese Unterschiede in der Beeinflussung der Meßergebnisse durch Lagerung könnten zum Teil durch eine erhöhte intrazelluläre Konversion bzw. Metabolisierung einzelner Steroide bedingt sein, wie in in-vitro-Versuchen mit Erythrocyten beim Testosteron, beim Androstendion $(8,9)$ und bei den Gestagenen $(25)$ sowie in-vivo bei den Östrogenen und Androgenen $(26,27,28)$ durch Messung der Konversion nachgewiesen wurde. Überraschend war die Zunahme von Cortisol im Vollblut bei Lagerung über 4 Tage. Möglicherweise läßt die Adsorption von Cortisol an Erythrocyten (6) im Laufe der vier Tage nach, oder intrazelluläres Cortisol $(8,29)$ wird freigesetzt.

Frühere Untersuchungen hatten gezeigt, daß einige Proteohormone $(5,15,30)$, und Bindungsproteine (4) bei Raumtemperatur instabil sind, so daß der größte Einfluß durch „präanalytische Störfaktoren“ bei den Bindungsproteinen erwartet wurde. Jedoch war der Anteil an freiem Cortisol und an freiem Testosteron in unseren Untersuchungen kaum verändert und zeigte sogar bei den Versuchen mit Plasmaproben oft geringere Abweichungen als es bei Messung der Gesamtsteroide der Fall war. Bei den Vollblutproben, die über 4 Tage bei $22^{\circ} \mathrm{C}$ gelagert hatten, findet sich jedoch sowohl für das freie Cortisol wie für das freie Testosteron eine erhebliche $(p<$ 0,01) Abnahme der Bindung. Bei dem Verfahren der Messung der freien Hormonanteile wird nicht unterschieden zwischen Bindung an SHBG, Transcortin und Albumin $(13,31)$. Es wurde deshalb geprüft, ob das spezifische Bindungsprotein ebenfalls durch Lagerung und Temperatur verändert wird. Wie die Messung von SHBG zeigt, ist selbst bei Lagerung unter Raumtemperatur über 4 Tage kein signifikanter Abfall eingetreten. Dies widerspricht einer früheren Mitteilung (4), wo jedoch vergleichbare systematische Untersuchungen nicht durchgeführt wurden. Die mangelnde inverse Korrelation zwischen SHBG und freiem Testosteron könnte durch die praktisch unbegrenzte Bindungskapazität von Albumin bedingt sein (13), so daß isolierte Verănderungen der Bindungsproteine verdeckt werden können (31).

Eine weitere Frage war, wie stabil Steroidkonjugate sind. Zu diesem Zweck wurde das Dehydroepiandrosteronsulfat untersucht. Auch hier: waren die Abweichungen $\mathrm{zu}$ den Kontrollwerten äußerst gering und, wie die Messung von Dehydroepianrosteron in einem Zusatzversuch zeigte, lag auch kein nennenswerter Abbau von Dehydroepiandrosteronsulfat zu Dehydroepiandrosteron vor. 
Da viele Steroide heute mittels käuflicher Kits ohne Chromatographie gemessen werden, sollte in einer Zusatzuntersuchung geklärt werden, ob mit dieser unspezifischeren Methode ähnliche Meßergebnisse gefunden werden. Bei dem hier verwendeten Kit waren die Testosteronkonzentrationen bemerkenswert stabil und differierten nicht wesentlich mit den Werten, die im spezifischeren Verfahren gemessen wurden.

Um Abweichungen in den Meßdaten spezifisch auf das gemessene Steroid beziehen zu können, wurden sie vor Analyse chromatographisch gereinigt. Wie die Untersuchungen mit Testosteron zeigen, ist dies nicht zwingend notwendig. In der Zwischenzeit wurde auch die Stabilität von Cortisol unter Verwendung der kompetitiven Proteinbindungsmethode ohne Chromatographie (32) untersucht. Soweit bisherige Lagerungs- und Auftauversuche mit Plasma (bis zu 2 Jahren) zeigen, ergeben sich mit dem spezifischeren, hier verwendeten Meßverfahren, vergleichbare Meßdaten.

Es stellt sich die Frage, ob die verwendeten Plasmaund Blutproben von Patienten mit Hämochromatose repräsentativ sind auch für die Gesunder oder von Patienten mit anderen Erkrankungen. Aufgrund ausgedehnter Vorversuche mit Plasma des Laborpersonals sowie mit Poolplasma, dem nach Charcoalvorbehandlung definierte Steroidmengen zugesetzt worden waren (unveröffentlicht), sind wir sicher, daß sich bei systematischer Untersuchung dieser Frage wesentliche Abweichungen von unseren Ergebnissen nicht finden werden.

Obwohl bei unseren Proben keine besondere Sorgfalt bezüglich Sterilität verwendet wurde, jedoch eine Reihe von Bakterien beschrieben worden sind, die in der Lage sind, Steroide zu metabolisieren (11, 12), sind je nach Verunreinigung der Proben unterschiedliche Ergebnisse denkbar.

Zusammenfassend kann aus unseren Untersuchungen für die Haltbarkeit von Steroiden und ihrer Bindung an Plasma-Proteine abgeleitet werden:

\section{Literatur}

1. Abraham, K. \& Rösler-Englhardt, A. (1978) Lab. Med. 2, A + B 133-137.

2. Guder, W. G. (1980) Internist $21,533-542$.

3. Koch, C. D. \& Rommel, K. (1978) Dtsch. Ärzteblatt 75, 1251-1252.

4. Wobser, E. (1971) Bindung von Testosteron und Ostradiol$17 \beta$ an Serumproteine bei gesunden Normalpersonen und bei Patienten mit Lebercirrhose. Promotionsarbeit, Univcrsität Bonn.
1. Wiederholtes Einfrieren und Auftauen von Steroiden beeinflußt die Meßergebnisse nicht, so daß zeitlich versetzte Mehrfachanalysen aus einer Plasmaprobe möglich sind.

2. Die Lagerung von Plasma bei $4^{\circ} \mathrm{C}$ (Kühlschrank) oder $22^{\circ} \mathrm{C}$ (Raumtemperatur) führt bei einigen Steroiden zu einem Abfall gegenüber den Kontrollen (Óstrogene, Aldosteron, Androstendion). Die Mittelwerte der Meßergebnisse liegen jedoch im 2SDBereich und bleiben deshalb in der Regel interpretierbar. Die Lagerung der Plasmaproben bei $4^{\circ} \mathrm{C}$ ergibt keine wesentliche Verbesserung.

3. Auch nach Lagerung der Proben als Vollblut waren die Abweichungen für die Messung der Gesamtsteroide meist nicht sehr groß und lagen oft im Bereich der gleichartig behandelten Plasmaproben. Sie betrugen jedoch bei Aldosteron und Androstendion mehr als $10 \%$.

4. Bemerkenswert stabil waren die Bindungen von Steroidhormonen an Plasmaproteine. Allein nach Lagerung von Vollblut bei Raumtemperatur über 4 Tage fanden sich deutliche und für eine sinnvolle Interpretation nicht mehr akzeptable Abweichungen für das freie Cortisol und das freie Testosteron.

5. Da die Abweichungen der Meßdaten von Steroiden bei den Untersuchungen innerhalb des 2SDBereiches lagen, scheint es erlaubt, Meßdaten von Steroiden auch dann klinisch zu interpretieren, wenn präanalytische Störfaktoren, wie Lagerung und Temperatur nicht exakt eliminiert wurden. Hiervon sind die Messungen der freien Steroide nach Lagerung als Vollblut jedoch ausgenommen.

\section{Danksagung}

Für ihre hervorragende technische Assistenz danken wir Frau Junker, Frl. Müller, Frau Pier und Frau Seifferl.
5. Yalow, R. S. \& Berson, S. A. (1960) J. Clin. Invest. 39, 1157-1175.

6. Bowman, R. E. (1975) In: Methoden der Hormonbestimmung (Breuer, H., Hamel, D. \& Krüskemper, H. L., eds.) Thieme Verlag, Stuttgart, pp. 183-189.

7. Eik-Nes, K. B. (1970) The androgens of the testis (Elk-Nes, K. B., ed.) Marcel Dekker, New York, p. 32, 54

8. Holzbauer, M. \& Vogt, M. (1961) J. Physiol. 157, 137-156. 
9. Kochakian, C. D. \& Arimasa, N. (1976) In: Anabolic androgenic steroids (Kochakian, C. D., ed.) Springer-Verlag, Berlin-Heidelberg-New York, pp. 314-317.

10. Lippmann, M. E. (1979) Glucocorticoid hormone action (Baxtcr, J. D. \& Rousseau, G. G., eds.) Springer-Verlag, Berlin-Heidelberg-New York, pp. 377-397.

11. Dorfman, R. I. \& Ungar, F. (1965) Metabolism of steroid hormones, Academic Press, New York, pp. 224-264.

12. Eppstein, S. H., Neister, P. D., Murray, H. C. \& Peterson, D. H. (1956) Vitamins and Hormones 14, 359-432.

13. Kley, H. K. \& Krüskemper. H. L. (1976) Med. Klin. 71, 799-805.

14. Baumann, J. E. (1982) Clin. Chem. 28, 2336-2337.

15. Kubasik, N. P., Ricotta, M., Hunter, T. \& Sine, H. E. (1982) Clin. Chem. 28, 164-165.

16. Wickings, E. J. \& Nieschlag, E. (1976) Clin. Chim. Acta 71, $439-443$.

17. Kley, H. K., Bartmann, E. \& Krüskemper, H. L. (1977) Acta Endocrinol. 85, 209-219.

18. Kley, H. K., Elsässer, W., Dehnen, H. \& Krüskemper, H. L. (1978) Steroids 32, 223-232.

19. Youssefnejadian, E.. Florensa, E., Collins, W. P. \& Sommerville, I. F. (1972) Steroids 20, 773-788.

20. Kley, H. K. (1975) Östrogene im Plasma des Mannes. Urban \& Schwarzenberg, München.

21. Nieschlag, E., Mauss, J., Coert, A. \& Kicivic, P. (1975) Acta Endocrinol. 79, 366-374.
22. v. Kammeren, E., Thijssen, J. H. H., Rademaker, B. \& Schwarz, F. (1975) Contraception 11, 53-59.

23. Kley, H. K., Peerenboom, H., Wagner, K. F. \& Krüskemper, H. L. (1982) In: The endocrines and the liver (Langer, M., Chiandussi, L., Chopra, I. J. \& Martini, L., eds.) Academic Press, London-New York, pp. 101-115.

24. Albert, J. P., Frịcke, G. \& Hartmann, F. (1977) Diagnostik 10, 719-720.

25. van der Molen, H. J. \& Groen, D. (1968) Acta Endocrinol. $58,419-444$.

26. Breuer, J. \& Breuer, H. (1973) Z. Klin. Chem. Klin. Biochem. 11, 263-269.

27. Gordon, G. G., Olivo, J., Rafii, R. \& Southren, L. (1975) J. Clin. Endocrinol. 40, 1018-1026.

28. Kley, H. K., Nieschlag, E., Wiegelmann, W. \& Krüskemper, H. L. (1975) Acta Endocrinol. 79, 275-285.

29. Bush, I. E. (1957) In: Hormones in blood (Wolstenholme, G. E. W. \& Millar, E. C. P., eds.) Ciba Found. Coll. Endocrinol. $11,263-285$.

30. Richards, J. B. \& Sayers, G. (1951) Proc. Soc. Exptl. Biol. Med. 77, 87-93.

31. Burke, C. W. \& Anderson, D. C. (1972) Nature (Lond.) 240, 38-40.

32. Kley, H. K. \& Krüskemper, H. L. (1971) Z. Klin. Chem. Klin. Biochem. 9, 520-526.

Prof. Dr. med. H. K. Kley

Medizinische Klinik C

Universität Düsseldorf

Moorenstraße 5

D-4000 Düsseldorf 